\section{Brunescence in the aging canine lens}

\section{David Williams}

Department of Veterinary Medicine, University of Cambridge, Cambridge, Cambs, UK

Brunescence, or lens browning, is a common finding in the ageing human lens but has not been reported to date in the dog. In the human brunescence in the aging lens correlates with the degree of protein thiolisation where photo-oxidation of sulphate groups yields disulphide bridges, a key step in age-related cataractogenesis which we know also occurs in the canine lens. Here the lenses of older dogs were examined to evaluate the amount of brunescence in the ageing canine lens.

20 lens from dogs of varying breeds and ages from 7 months to 13 years, with a mean age of $7.3 \pm 4.0$ years were evaluated. Lenses were removed from eyes at post-mortem from dogs with eyes which, on ophthalmological examination pre-mortem, were unremarkable, barring nuclear sclerosis in dogs over 9 years of age but without overt cataract. Lenses were photographed against a white illuminated background and against a grid of lines. The resulted images were analysed using the NIH image analysis system Image $\mathrm{J}$ to determine the degree of light absorption in the nucleus and cortex. Section of lens were subject to microspectrophotometric analysis using the CamSpec spectrophotometer 105 (Spectronic Camspec Ltd). Absorption of light of different wavelengths by the nucleus and cortex was correlated with age of the dog.

Examination of the ageing canine lens showed obvious brunescence as the dog ages. Image analysis of photographic images showed a clear disparity between light absorption in the nucleus and the cortex, with significantly more absorption in the nucleus. Spectrophotometric evaluation showed a greater absorption of light at lower wavelengths in the ageing lens and greater transmission of light in longer wavelengths, giving the brown appearance to the lens. Visualisation of grid lines through the lens became significantly more difficult as the lens aged although quantifying this gradual reduction in lens clarity numerically was difficult.

Given that dogs cannot tell us of the colour disparity of their vision as they age, as humans do, little attention has been paid to vision in older dogs without overt cataract. Here by evaluation of lenses post-mortem, it was clear both that lens clarity reduces even without the appearance of overt cataract, and that the ageing lens undergoes substantial brunescence. The relevance of this to vision in the ageing canine patent still awaits further study.

\section{Efficacy of a cross-linked} hyaluronic acid (HA) polymer on increasing healing rates of feline stromal corneal ulceration compared with a HA tear replacement drop: results of a masked controlled study

\section{David Williams}

Department of Veterinary Medicine, University of Cambridge, Cambridge, Cambs, UK

Topical hyaluronic acid (HA) gels have been widely used as a tear replacement but the beneficial effects of $\mathrm{HA}$ as a ligand for the CD44 cell surface receptor with regard to cell migration and adhesion suggest that a formulation with a prolonged corneal residence time may be valuable in promoting the healing of corneal ulcers. A cross-linked HA product (xCMHA-S: (Remend Bayer) has been shown to stimulate corneal ulcer healing in an experimental rabbit model. Here we seek to ask whether topical XCMHA-S promotes healing of feline stromal ulceration more than a standard HA tear replacement drop in a masked controlled study.

30 cats affected with a stromal ulcer were included in the study. The animals were subject to a full systemic and ophthalmic examination and those with concurrent ocular or systemic disease were excluded from the study. Cats were randomly assigned to be treated with $\times \mathrm{CMHA}-\mathrm{S}$ or HA tear replacement drop (HATRD) three times daily together with topical antibiotic applied concurrently. The identity of the $\mathrm{xCMHA}-\mathrm{S}$ or HATRD was masked since the product was identified only with a code number. Informed consent was provided by the owner. Re-examinations of each animal were conducted weekly until failure of the ocular surface to stain with fluorescein dye demonstrated ulcer healing. Time to ulcer healing was compared using an unpaired Students $T$ test.

One cat was lost to follow up leaving 29 cats which fulfilled the trial, 15 animals treated with $\times C M H A-S$ and 14 with HATRD. The average age of the $x \mathrm{CMHA}$-S-treated cats was $9.3 \pm 3.7$ years while the age of of the HATRD-treated cats was $8.6 \pm 2.1$ years, these not significantly different at $p=0.51$. Eight of the ulcers treated with $\times \mathrm{CMHA}-\mathrm{S}$ were mid-stromal in depth with three being superficial stromal and four deep stromal. Of the HATRD-treated ulcers 13 were mid-stromal and one was deep stromal. The time to ulcer healing with XCMHA-S was $21 \pm 11$ days and the time to healing with HATRD was $31.8 \pm 10.3$ days, this significantly longer than for the $x C M H A-S$ group at $p=0.011$.

This small study has shown that $x$ CMHA-S reduces the healing time for feline stromal corneal ulceration by an average of 10 days. We suggest that the product can be a viable treatment for feline corneal stromal ulcers. 